\title{
Caracterizaciones contemporáneas de la cultura: metáforas materialistas e idealistas acerca del patrimonio cultural en textos online de $L a$ Nación, Clarín y Página/12
}

\author{
Felicitas Casillo \\ Facultad de Comunicación, Universidad Austral. \\ fcasillo@austral.edu.ar \\ Fecha de finalización del trabajo: 27 de enero de 2020. \\ Recibido: 15 de julio de 2020 \\ Aceptado: 27 de noviembre de 2020 \\ DOI: https://doi.org/10.26422/aucom.2020.0902.cas
}

\section{Resumen}

La presente investigación se focalizó en el discurso mediático acerca de procesos de transformación del patrimonio cultural. En esos discursos pueden hallarse al menos algunas de las principales definiciones contemporáneas sobre la cultura. El estudio de caso fue el relato de la transformación institucional y remodelación edilicia del Teatro Colón, en la versión online de los diarios La Nación, Clarín y Página/12, entre 2006 y 2011.

La metaforización funcionó como un mecanismo de representación de la cultura a partir del cual se concretan diversas caracterizaciones. Estas fueron organizadas mediante la familia metafórica económica y la familia metafórica religiosa. A partir de un enfoque hermenéutico, se concluye que una caracterización válida de cultura será aquella que albergue a los protagonistas de una tradición cultural.

Palabras clave: hermenéutica, cultura, discurso, patrimonio, metáfora, performatividad.

Contemporary definitions of culture: materialistic and idealistic metaphors of cultural heritage in online articles for La Nación, Clarín, and Página/12

\begin{abstract}
Our investigation focuses on the media discourse surrounding the transformation of cultural heritage. Embedded in this discourse, one can find some of the leading contemporary definitions of culture. For our case study, we looked at how the digital editions of three major newspapers in Argentina - La Nación, Clarín, and Página/12 - covered the institutional transformation and remodeling of the Colón Theater in Buenos Aires from 2006 to 2011.
\end{abstract}




\begin{abstract}
Throughout this media coverage, metaphors served to represent and characterize culture. We organized these in two families: economic and religious metaphors. Via a hermeneutical approach, we conclude that a valid characterization of culture is one that can house and contain the protagonists of a cultural tradition. Keywords: hermeneutics, culture, discourse, heritage, metaphor, performativity.
\end{abstract}

\title{
Caracterizações contemporâneas da cultura: metáforas materialistas e idealistas sobre o patrimônio cultural em textos online de $L a$ Nación, Clarín e Página / 12
}

\section{Resumo}

A presente pesquisa concentrou-se no discurso da mídia sobre processos de transformação do patrimônio cultural. Pelo menos algumas das principais definições contemporâneas de cultura podem ser encontradas nesses discursos. O estudo de caso foi a narração da transformação institucional e remodelação predial do Teatro Colón na versão online dos jornais La Nación, Clarín e Página / 12, entre 2006 e 2011.

A metaforização funcionou como um mecanismo de representação da cultura, a partir da qual várias caracterizações são especificadas. As caracterizações culturais encontradas no caso foram organizadas através da família metafórica econômica e da família metafórica religiosa. A partir de uma abordagem hermenêutica, conclui-se que uma caracterização cultural válida será aquela que ofereca abrigo aos protagonistas de uma tradição cultural.

Palavras-chave: hermenêutica, cultura, discurso, patrimônio, metáfora, performatividade.

\section{Introducción}

Las definiciones de "cultura" fueron un asunto medular para diversos sistemas filosóficos, enfoques epistemológicos y corrientes políticas: la cultura como perfección de la naturaleza; la cultura frente a la civilización; la cultura como concreción del espíritu; la cultura como reflejo de las condiciones de producción; la cultura como parte de esas condiciones de producción y como proceso de preparación para la revolución; la cultura como la totalidad de creaciones y artefactos humanos; la cultura como condición del statu quo; la cultura como parte de la industria, entre otras.

Este artículo se propone, por un lado, exponer una síntesis de diversos aportes históricos acerca del término "cultura" y, por el otro lado, describir los resultados de una investigación acerca de caracterizaciones relevantes en la actualidad. Estas definiciones fueron halladas en la versión online de tres diarios argentinos. Los textos analizados pertenecieron a diversos géneros: noticias, editoriales, entrevistas y cartas de lectores, entre otros.

El valor de esta indagación radica, entonces, en averiguar cuál es el significado adjudicado al término o realidad a la que se denomina "cultura". Este hallazgo, que podría servir para el diseño de hipótesis en futuras investigaciones, no es generalizable, sino que proviene de un estudio de caso relacionado al patrimonio cultural. La elección del 
Austral Comunicación

Volumen 9, número 2 (Diciembre de 2020): 159-186. ISSN 2313-9129

discurso acerca del patrimonio cultural permite circunscribir el enorme campo de la cultura y posibilita también indagar acerca de las caracterizaciones existentes sobre instituciones o expresiones ligadas al pasado o a la tradición.

Para hallar esta descripción, es necesario observar los juegos del lenguaje -en términos de Ludwig Wittgenstein (1953)- en los que la palabra asume significación. En este sentido, la noción de "discurso" se aleja de un enfoque únicamente referencial. Es decir, el discurso de la cultura no solo es palabra sobre cultura, sino, sobre todo, aquella comunicación en la que la cultura sucede y tiene sentido en el mundo de la vida de sus protagonistas. Por esta razón, la significación del término tiene consecuencias pragmáticas, por ejemplo, en la gestión cultural, en las políticas culturales y también en el modo en el que las personas conciben la cultura, tanto individualmente como de modo comunitario.

Este discurso de la cultura, sin embargo, es amplio. Incluso podría describírselo, en palabras de Marc Angenot (2010), como parte de un discurso inabarcable por su anchura, el "vasto rumor" de una época (p. 22). Es por eso que debe circunscribirse el objeto de estudio. Seguramente existan otras caracterizaciones actuales en otro tipo de discursos no mediáticos y sobre otro tipo de objetos culturales. En ese sentido, más que exhaustividad, esta investigación se propuso la construcción de una hipótesis sobre un tema que tiene enormes consecuencias en la construcción del sentido personal y en la identidad y la memoria de las comunidades.

Como se observará a continuación, desde el mismo origen, el término "cultura" fue metafórico. Es decir, se concibió la cultura como algo que era otra cosa o, al menos, algo que era como otra cosa. La idea de cultura -amplia y, por momentos, inasible-pareció necesitar de metáforas para ser comprendida. El marco teórico de este artículo se propone subrayar sobre todo dos grandes grupos de definiciones modernas: las idealistas y las materialistas. Luego, se expondrá el hallazgo de caracterizaciones actuales que guardan relación con esos enfoques históricos.

Con respecto a las reflexiones acerca de la metáfora, estas se inauguran con la Poética de Aristóteles (1948), para quien la metáfora era la más grande de las figuras retóricas, "pues aplicar bien las metáforas es indagar qué cosas son entre sí semejantes" (p. 48). A partir de aquel origen vinculado a la retórica clásica, y luego de una etapa ligada a una retórica limitada a la clasificación de tropos, desde la segunda mitad del siglo XX en adelante diversos autores han estudiado la metáfora como mecanismo textual de producción de sentido, que excede el uso literario: Fauconnier (1997), Gibbs (1994), Glucksberg (2001), Grady, Oakley y Coulson (1999), Johnson (1981), Kövecses (2002), Lakoff (1987, 1994, 2002), Lakoff y Johnson (2009), Lakoff y Turner (1989), Ricoeur (2001), Searle (1979) y Semino (1997), entre otros. 
Felicitas Casillo

Caracterizaciones contemporáneas de la cultura: metáforas materialistas e idealistas acerca

del patrimonio cultural en textos online de La Nación, Clarín y Página/12

\section{La historia de una definición}

Fue a partir de la Ilustración cuando la filosofía concibió a la cultura como concepto diferencial.Anteriormente, existían referencias a una realidad semejante a la cultura, pero aún no se la concebía con ese término. De esas alusiones anteriores, pueden destacarse, entre otras, el concepto platónico-aristotélico y estoico de æon, unidad fundamental en la vida de la naturaleza y en la vida moral del hombre, y la caracterización de Cicerón: cultura animi philosophia est, "la filosofía es el cultivo del espíritu"(Dempf, 1933; Kroeber y Kluckhohn, 1952). También durante el Medioevo se valorizó la idea de invención, en el sentido de que toda utilidad debía concebirse con esplendor (Pernoud, 2010).

A partir del siglo XVIII, existieron dos enfoques teóricos sobre la cultura que se mantuvieron durante la modernidad: una interpretación idealista y otra materialista. Es decir, desde el punto de vista metafórico, cultura como espíritu o cultura como materia. Ambas visiones tienen la pretensión de construir toda la filosofía de la cultura bajo el primado de un factor determinante. Ernst Gombrich (2015), al igual que Alois Dempf (1933), identificaba en esta dualidad el "pecado original" de los estudios sobre la cultura.

La concepción idealista primó desde el XVIII hasta el inicio del siglo XIX, mientras que luego tuvo mayor gravitación una visión economicista. El problema de las concepciones modernas de la cultura será, entonces, la cuestión de qué factor determina el movimiento y la realidad cultural. La visión idealista se basaba en la idea hegeliana de cultura como concreción o reflejo del espíritu. En la visión marxiana, también determinista, el espíritu abandonaba su condición de eje y en su lugar se ubicaban los factores de producción. Las actividades que antes se encontraban en la periferia de la rueda, cuyo centro era el espíritu, pasaban a ser "superestructura", reflejo de esa base. El materialismo histórico introdujo una novedad particular: de la visión esotérica de Hegel se pasó a una concepción materialista.

A pesar de la crítica a la tesis marxista realizada por Antonio Gramsci (2017) y por otros teóricos dentro de ese paradigma, el marxismo redujo el diálogo sobre la cultura a la discusión sobre las condiciones de producción. A partir de esta definición, Gramsci (2017) volvió a acercarse a una corriente hegeliana de la historia, en tanto subrayó el lugar del "espíritu". El desarrollo de la cultura, decía, era paulatino: “[...] toda revolución ha sido precedida por un intenso trabajo de crítica, de penetración cultural, de permeación de ideas [...]" (p. 18).

\section{Actualidad de las caracterizaciones históricas}

Baldwin, Faulkner, Hecht y Lindsley (2008), Eagleton (1999, 2000), García Canclini $(1987,1997,1989,2010,2012)$ y Williams $(1953,1981)$ coinciden en que existen otras tres categorías más concretas en el uso de esta palabra, además del enfoque idealista y materialista. La primera, desde el siglo XVIII, se refiere al "cultivo" en términos de 
desarrollo intelectual, espiritual y estético; ese sentido fue originalmente metafórico a partir del uso de "cultivo rural". Esta definición es en parte tributaria a la concepción idealista, aunque no siempre asumió los presupuestos ontológicos del idealismo. Algunos de los autores más influyentes de esta corriente son Johann Gottfried Herder, quien con el movimiento Sturm und Drang impulsó la visión romántica alemana, Matthew Arnold (2010) y, posteriormente y a su modo, el poeta T. S. Eliot (1948), entre otros. De Arnold (2010) es célebre una definición: "La cultura es la búsqueda de nuestra perfección completa [...] lo mejor que se ha pensado y dicho en el mundo [...]" (p. 41), y luego "[...] la cultura nos insta a concebir una armoniosa perfección, una perfección en la que los rasgos de la belleza y la inteligencia están presentes" (p. 85).

El segundo uso de la palabra "cultura", la definición "antropológica", se refiere a una forma de vida general. Esta noción amplia fue acuñada en 1871 por Edward Burnett Tylor y consistía en la totalidad de capacidades y hábitos adquiridos por el ser humano como miembro de la sociedad. Finalmente, existe un tercer uso que se circunscribe a las obras y prácticas intelectuales y, sobre todo, a la actividad artística.

Este término, entonces, ha funcionado como una especie de palabra síntesis que alude a diversas significaciones. Sobre esta polisemia, Baldwin et al. (2008) también alertaban acerca del riesgo de que el signo se transformara en "una vasija vacía esperando por personas -académicos y hablantes comunes- para que la llenen con significado" (p. 4).

Debe sumarse al anterior panorama la síntesis que Raymond Williams (1981), dentro de la Escuela de Birmingham, realizó del derrotero del concepto de "cultura". Con el materialismo marxista, Williams coincidía en que existía una estructura social global, pero se diferenciaba de este porque reconocía la existencia de una "producción cultural" o "práctica cultural" no como reflejo de la estructura, sino como elemento constitutivo de ella. De la definición antropológica, Williams (1981) toma el sentido más general. Esta nueva definición, de evidente inspiración gramsciana, en lugar de sostener la existencia de un "espíritu conformador", define a la cultura como el "sistema significante a través del cual un orden social se comunica, se produce, experimenta e investiga" (p. 13). En este sentido, el arte pasa a ser una de aquellas prácticas significantes, entre otras.

García Canclini (1989) prefirió una "definición restringida de cultura": "[la cultura implicaría] el conjunto de fenómenos que mediante la representación o reelaboración simbólica de las estructuras materiales contribuyen a reproducir o transformar el sistema social" (p. 14). El autor subraya que existe una organización material propia para cada producción cultural y en esto coincide con el concepto de "campo" de Pierre Bourdieu (2010). Luego, sigue: "El análisis de estas instituciones, de las condiciones sociales que establecen para la existencia de los productos culturales, es decisivo para la interpretación de dichos productos" (García Canclini, 1989, p. 20). 
Felicitas Casillo

Caracterizaciones contemporáneas de la cultura: metáforas materialistas e idealistas acerca

del patrimonio cultural en textos online de La Nación, Clarín y Página/12

El rol fundamental que ha tenido la comunicación en la historia contemporánea de la cultura se refleja, controversialmente, en el término "industria cultural", que fue un concepto clave de la teoría crítica y que tuvo originalmente un significado negativo. En la obra Dialéctica de la Ilustración, Adorno y Horkheimer (1998) se preguntaron cuál era la razón de la declinación cultural y, en esa obra, le dedicaron un duro capítulo a las industrias culturales. Dentro mismo de la Escuela de Frankfurt hubo un debate acerca de la naturaleza de la industria cultural. En 1936, Walter Benjamin (2015) terminaba La obra de arte en la era de su reproductibilidad técnica, una tesis en torno al arte y la tecnología que signó los estudios posteriores del arte y la estética en general. Benjamin reconocía cierta ventaja democratizadora en la industria. El autor definía la "autenticidad" de la obra de arte como "aura": una "distancia,"'la manifestación irrepetible de una lejanía" (Benjamin, 2015, p.34).La reproductibilidad rompía el aura porque quitaba a la obra "del dominio de la tradición" (p. 31). Pero, al mismo tiempo, cuando se refiere al cine, por ejemplo, concede: "Voló por los aires este mundo-prisión con la dinamita de una décima de segundo, de manera que ahora, en medio de sus vastas ruinas y escombros, emprendemos la travesía con calma y sed de aventura" (p. 55).

En este contexto, para Renato Rosaldo (2008), el concepto de "cultura" ha migrado a diferentes campos de estudio: "en la década de 1970, la cultura cambió de ser un objeto de estudio a convertirse [...] en una herramienta flexible para estudiar al servicio de diferentes proyectos analíticos. [...] se convirtió en una herramienta" (p. 11). Según Baldwin et al. (2008), en las últimas décadas del siglo XX han aparecido definiciones de "cultura" que se centran en su condición de sistema simbólico. Estas consideraciones se relacionan con las llamadas "definiciones posmodernas de cultura", en las que la noción de "discurso" cobra mayor relevancia (p. 21).

Para Gustav Jahoda (2012), la diversidad que dispara el término "cultura" se ha multiplicado. El autor considera que, en muchos casos, esas interpretaciones son contrapuestas

es un concepto todavía indispensable. La mayor parte del tiempo se lo utiliza sin definirlo [...]. Sin embargo, por razones teóricas y empíricas, es esencial que los autores detallen el sentido específico al que se refieren cuando utilizan el término cultura (p. 300).

El problema de la cultura analizada exclusivamente desde una dimensión interior o únicamente como materialidad es tratado también por Irina Mironenko y Pavel Sorokin (2018), quienes retoman el clásico problema de la diversidad de definiciones, pero no parecen coincidir con Jahoda en la probabilidad de enunciar una definición única a partir de visiones parciales.

\section{Patrimonio cultural: de la performatividad a la interpretación}

Entre quienes desarrollan el campo interdisciplinar de las teorías sobre la cultura, varios autores subrayan el sitio crucial que ocupa el patrimonio cultural y la cultura 
Austral Comunicación

Volumen 9, número 2 (Diciembre de 2020): 159-186. ISSN 2313-9129

en las sociedades contemporáneas en relación con la memoria, con la identidad, pero también con el turismo y con las industrias creativas (Ballart, 1997; García Canclini, 1999; Groys, 2008; Lipovetsky y Serroy, 2017; Resch y Steinert, 2003). El enfoque teórico que concibe a la cultura como un recurso (Buitrago Restrepo y Duque Márquez, 2013; Dormaels, 2011; Du Gay y Pryke, 2002; Hopkins, 2013; Yúdice, 2002) suele subrayar sobre todo el carácter performativo de la cultura y del patrimonio, es decir, la cultura y el patrimonio como exclusivas construcciones simbólicas o discursivas.

Dentro de los estudios acerca de la cultura, la noción de "performatividad" ha ganado protagonismo en las últimas décadas. La consideración del patrimonio cultural y de la cultura exclusivamente como construcciones discursivas ${ }^{1}$ podría redundar en una visión peyorativa de la materialidad, propia de visiones románticas. La idea de que el objeto patrimonial es meramente soporte de significación parece responder a una etapa germinal en los estudios de la comunicación, ligada al modelo informacional para el cual el desafío era exclusivamente la transmisión y no consideraba, por ejemplo, la riqueza de la interpretación. Esta idea limitada del signo fue compartida, curiosamente, por la visión positivista que inauguró el giro lingüístico del siglo XX: la del signo como pura referencialidad. Desde la perspectiva de la performatividad, el signo-objeto patrimonial se constituye por su significación, pero, al mismo tiempo, esta significación es variable, y surge entonces la pregunta acerca de la "legitimidad" de los nuevos sentidos adjudicados (Steiner, 1993). Esta legitimidad, que es crucial en cualquier proceso de interpretación, lo es, a su vez, en la comprensión del patrimonio, que se trata también de un proceso de comprensión entre personas.

La consideración discursiva implicaría ciertamente subrayar la comunicación alrededor del patrimonio como un elemento crucial de la cultura (García Canclini, 1999; Groys, 2008). Las diversas significaciones que se superponen sobre un objeto patrimonial lo transforman en un palimpsesto, sin que por eso deba dejar de ser lo que es, aquello que lo define y que podría hallarse en tanto se intentara el diálogo con su horizonte de sentido original. Con respecto a esto, la hermenéutica del arte y la cultura ha subrayado la naturaleza simbólica de la obra, la obra como el sitio de encuentro y comprensión entre, al menos, dos intérpretes (Ablett y Dyer, 2009; Gadamer, 1996; Morisset, 2011; Steiner, 1993; Tilden, 1977).

\section{Metodología}

El análisis de los textos que integran el corpus se concretó a partir de una metodología de lectura hermenéutica, que consistió en diversas estrategias de lectura sobre

En términos de John Austin (1962) -de quien esta teoría ha tomado el concepto-, un acto performativo existe a partir de su enunciación. Sin embargo, la acción que concreta un performativo perdura más allá de la enunciación. 
Felicitas Casillo

Caracterizaciones contemporáneas de la cultura: metáforas materialistas e idealistas acerca

del patrimonio cultural en textos online de La Nación, Clarín y Página/12

un mismo conjunto de textos. Este se conformó a partir de la búsqueda de palabras clave en la web de cada diario. Algunos de estos términos fueron, por ejemplo: "Teatro Colón" y "patrimonio cultural". Es decir, la primera etapa de lectura se realizó en el "sitio" de hallazgo de cada texto.

Luego, por medio del uso de una unidad hermenéutica en el software ATLAS.ti, se procedió a cargar el conjunto del corpus de los tres medios. La etapa siguiente fue una fase exploratoria de estadística textual.Los índices de ocurrencias de cada término facilitaron la búsqueda de posibles variables, que luego se observaron mediante estrategias de análisis del discurso. Esta "lectura cuantitativa", sin embargo, fue tan solo un sondeo, que si bien permitió observar la aparición de actores y de campos semánticos, fue superado luego por la etapa cualitativa.

A partir de entonces, se redactó la historia del conflicto y se dividió el relato en "hitos". Luego, se seleccionaron del corpus aquellos textos que fueran más significativos para cada hito (ver Tabla 1), esto es, que retrataran con mayor precisión qué sucedía en cada momento del conflicto o que las fuentes citadas o los acontecimientos narrados fueran de importancia para comprender lo ocurrido. Algunas de las categorías que comenzaron a relevarse en un principio en el ATLAS.ti de forma inductiva luego se abandonaron y adquirió mayor relevancia la observación de la categoría emergente "metáforas". A partir de entonces, se extrajeron los párrafos con términos o enunciados metafóricos; luego, se seleccionaron casos de funcionamiento metafórico familiar y se describieron de forma minuciosa. Finalmente, se procedió a interpretar dichos hallazgos.

Tabla 1. Cantidad de textos que integraron el corpus de análisis ${ }^{2}$

\begin{tabular}{ll}
\hline Diario & Total de textos a partir de la recolección por palabras clave \\
\hline Muestra Teatro Colón (2006-2011) La Nación & 305 \\
\hline Muestra Teatro Colón (2006-2011) Clarín & 227 \\
\hline Muestra Teatro Colón (2006-2011) Página 12 & 128 \\
\hline Corpus total & 660 \\
\hline Corpus total a partir de la selección por hitos & 461 \\
\hline
\end{tabular}

\section{Problema o pregunta inicial}

El tema estudiado fue el discurso mediático sobre instituciones ligadas al patrimonio cultural durante procesos de transformación edilicia y/o institucional. De estos

2 Todas las tablas son de elaboración propia. 
conflictos, se seleccionó para la presente investigación el proceso de restauración del Teatro Colón y su transformación en un ente autárquico (2006-2011). ${ }^{3}$

Pregunta inicial: ¿cuál es la caracterización del patrimonio cultural y de la cultura que se concreta en la versión online de los diarios La Nación, Clarín y Página/12 durante procesos de crisis en instituciones culturales?

Objetivo general: analizar qué caracterización del patrimonio y de la cultura se realiza a lo largo de la cobertura de los acontecimientos noticiosos ligados al patrimonio cultural en la versión online de los diarios La Nación, Clarín y Página/12 durante etapas de crisis en instituciones culturales.

\section{Estudio de caso y justificación}

El caso analizado fue la cobertura mediática de la restauración y reforma institucional del Teatro Colón, entre 2006 y 2011, en la versión digital de los diarios La Nación, Clarín y Página/12. A pesar de que la precarización laboral y el deterioro edilicio habían comenzado hacía décadas, y aun cuando el plan de remodelación y rescate patrimonial, llamado "máster plan", se había iniciado en 2001, la investigación se enfocó en el periodo mencionado porque durante ese tiempo el conflicto escaló y alcanzó su desenlace. Se partió del supuesto de que los medios aún son un actor relevante en la construcción de sentido social, sobre todo en noticias que no son protagónicas en la agenda y, por ende, no tienen mayor repercusión en otros ámbitos discursivos, por ejemplo, las redes sociales.

El conflicto del Teatro Colón se desarrolló en un marco histórico singular: el Bicentenario de la República Argentina. Se observa una pugna entre dos visiones de la nación y, de modo más general, dos tendencias contrapuestas en Sudamérica, representadas en este caso por los oficialismos, las dos fuerzas políticas preponderantes en esta encrucijada. Por un lado, el Frente para la Victoria, desde la Presidencia de la Nación; por el otro, el partido Propuesta Republicana (PRO), desde el Gobierno de la Ciudad de Buenos Aires. El Teatro Colón fue uno de los símbolos de las celebraciones del Bicentenario y una institución cuya representación se disputaron estas fuerzas políticas.

\section{Técnica de observación}

\section{Conformación del corpus}

Población: los textos analizados pertenecen a la versión online de los diarios $\mathrm{La}$ Nación, Clarín y Página/12. El periodo estudiado fue desde 2006 hasta 2011.

\footnotetext{
La investigación original incluyó también un caso menor de contraste: la transformación del edificio del Correo Central de Buenos Aires en el Centro Cultural Néstor Kirchner (2015). Este caso, sin embargo, no se expone en el presente artículo.
} 
Felicitas Casillo

Caracterizaciones contemporáneas de la cultura: metáforas materialistas e idealistas acerca

del patrimonio cultural en textos online de La Nación, Clarín y Página/12

Muestra: fue no probabilística estratégica, también llamada "intencional o teórica", y se conformó a partir de una selección por hitos temporales según las diversas etapas del conflicto. La unidad de análisis fue el texto (noticia, editorial, entrevista, carta de lectores y breve, entre otros). La distinción del género no fue una categoría relevante para el objetivo de esta investigación.

La metodología de la lectura hermenéutica diseñada para este estudio fue indispensable debido a la extensión del corpus. A partir de diversas etapas de lectura, se buscó identificar aquellos mecanismos relevantes de producción de sentido en el conjunto de textos. Luego de una etapa exploratoria, se observó que la metáfora funcionaba como una estrategia textual que vinculaba el contexto propio de los protagonistas de la institución patrimonial con otros contextos diversos, y que este proceso redundaba en una representación particular del patrimonio cultural y de la cultura en general. El método de análisis del discurso diseñado para esta investigación se inspiró en los trabajos de Damián Fernández Pedemonte $(1996,1999,2011)$ e Irene Vasilachis de Gialdino (2006).

El esfuerzo por comprender la importancia del lenguaje en los mundos de la vida, en los que se halla el patrimonio cultural y se desarrolla su gestión, y de concebir los campos culturales como confluencias discursivas se concreta en la práctica de la metodología cualitativa del análisis del discurso. A través de ciertas estrategias de metaforización, es posible explicar la influencia que tienen todavía algunas corrientes teóricas sobre las caracterizaciones de cultura más influyentes en el ámbito público mediático. Es decir, la cuestión paradigmática implica el mismo problema de investigación.

\section{Relevamiento}

Durante la etapa de lectura del corpus se relevaron diferentes metáforas, que funcionaron como mecanismos de representación a partir de la acción metaforizadora. La focalización del presente estudio en la metáfora no significa que no aparezcan otros juegos de lenguaje en el corpus, sino que, de los observados, este fue el que reveló la construcción de sentido a partir de una caracterización idealista o materialista del patrimonio y la cultura. Las metáforas halladas pudieron agruparse según su semejanza temática, que redundó en una coherencia entre diferentes niveles de metáforas ubicados en un mismo dominio o gestalt, en términos de Lakoff y Johnson (2009).

Se denominó "familia metafórica", entonces, a un grupo de metáforas que mantienen entre sí vínculos de coherencia temática. Cada familia está conformada por niveles que, si bien son diversos, mantienen relaciones de coherencia y de semejanza. El término "familia metafórica" fue inspirado en el "parecido de familia" -familienähnlichkeit, en alemán- que observaba Ludwig Wittgenstein (1953, p. 32) entre ciertos juegos del lenguaje. 
Austral Comunicación

Volumen 9, número 2 (Diciembre de 2020): 159-186. ISSN 2313-9129

El término "familia", entonces, posee un significado también metafórico y describe la existencia de redes de figuras que pertenecen a un mismo dominio, que mantienen lazos internos de coherencia y que pueden "emparentarse" con otros niveles de la misma familia o de otras familias. El hallazgo de una familia metafórica en un corpus implica que la metaforización, en lugar de ser ocasional, es amplia y compromete a varios actores, porque existen diversos niveles en los que se la desarrolla. El vínculo de coherencia en el interior de la familia, al menos en la presente investigación, fue temático. Este vínculo puede ser, a su vez, de complementariedad o antagonismo.

Dos fueron las familias metafóricas más relevantes relacionadas al patrimonio cultural y a la cultura: la "familia económica" y la "familia religiosa" (ver Tabla 2). Estos grupos se caracterizaron por desarrollar una metáfora estructural a partir de diversos niveles. Para Lakoff y Johnson (2009), las metáforas estructurales son aquellas que producen una significación compleja y consisten en la estructuración metafórica de un concepto en términos de otro: permiten utilizar "un concepto muy estructurado y claramente delineado para estructurar otro" (p. 101). Un ejemplo de este tipo es "la política es una guerra", en el que la experiencia "guerra", conocida directa o indirectamente, permite una rápida comprensión de la realidad "política".

Tabla 2. Familias metafóricas con sus respectivos niveles internos

\begin{tabular}{ll}
\hline Familia económica & Familias metafóricas \\
\hline & Nivel recurso: "El patrimonio o la cultura son un recurso". \\
\hline & Nivel trabajo: "La cultura es trabajo". \\
\hline Familia religiosa & Nivel celebración: "La sumbolo: "El pattura es celebraciónonio o la cultura son un símbolo". \\
\cline { 2 - 2 } & Nivel templo: "La institución cultural es un templo". \\
\hline & Nivel sacerdote: "El artista es un sacerdote". \\
\hline
\end{tabular}

En la primera de las familias metafóricas, el eje central que estructura la red de metáforas es la economía, y luego se concreta en los niveles "patrimonio o cultura son recurso" y "cultura es trabajo". En la segunda de las familias, el eje es la antigua concepción de la cultura como un sucedáneo religioso y se concreta en los niveles metafóricos "cultura es celebración", "la institución patrimonial o la cultura son un símbolo", "la institución patrimonial es un templo" y, aunque más débil, "el artista es un sacerdote".

Las dos grandes familias metafóricas que serán descritas a continuación son tributarias a las dos perspectivas presentadas en el marco teórico: el enfoque idealista y el materialista. Además, se observaron en el corpus otras metáforas que también se 
Felicitas Casillo

Caracterizaciones contemporáneas de la cultura: metáforas materialistas e idealistas acerca

del patrimonio cultural en textos online de La Nación, Clarín y Página/12

referían al campo cultural o a alguno de sus elementos o fenómenos. Un grupo de figuras muy común en el discurso de preservación sobre el patrimonio cultural son aquellas metáforas asociadas al riesgo: la "batalla" por el Teatro, el Teatro se "derrumba", el Teatro está "enfermo", etcétera. Luego, también existieron metáforas cuya falta de redundancia en el corpus o su completa cristalización les quitó relevancia.

\section{Proceso de estructuración de dominio resultante}

La acción de las metáforas estructurales consiste en estructurar un dominio -como por ejemplo, el campo cultural- a partir del dominio de una metáfora. Para facilitar la referencia, se llamó al dominio del campo cultural "estructura de dominio original" (EDO); y al dominio de la metáfora, "estructura de dominio metafórico" (EDM). La estructuración que produce EDM en la EDO se denominó "estructura de dominio resultante" (EDR). Este fenómeno de estructuración metafórica fue relevado a nivel discursivo y no a nivel cognitivo, como originalmente había sido estudiado por Lakoff y Johnson (2009).

En el caso de la familia económica, la EDM pertenece al nivel recurso y trabajo; en el caso de la familia religiosa, a los niveles celebración, símbolo, templo y sacerdote. El funcionamiento de las metáforas implica que algunos elementos de los dominios aparezcan destacados, enfocados o desenfocados u ocultos. Por ejemplo, el nivel trabajo destaca la labor artística como un empleo, pero desenfoca la formación artística y oculta las motivaciones no económicas, la formación y la creatividad.

La presente investigación se centró en el funcionamiento discursivo de las estructuras de dominio metafórico y la estructura de dominio resultante, en tanto estas fueron realidades textuales. Sin embargo, el lector no encontrará la descripción de la estructura de dominio original porque no se estudió el discurso original de los artistas ni se realizó observación participante en sus lugares de trabajo. El ejemplo de la Tabla 3 incluye algunos elementos solamente supuestos de la EDO.

Tabla 3. Ejemplo de proceso de estructuración de dominio resultante (EDR)

\begin{tabular}{ll}
\hline Elementos de la estructura de dominio original (EDO) & Elementos de la estructura de dominio metafórico (EDM) Nivel Trabajo \\
\hline Trabajo de los artistas (enfocado). & $\begin{array}{l}\text { Trabajo en general - jubilaciones, derechos, regímenes } \\
\text { laborales, etcétera - (enfocado). }\end{array}$ \\
\hline Creatividad (oculto). & Relación con el poder (enfocado). \\
\hline Motivaciones no económicas (oculto). & Relación entre los trabajadores -agremiación- (enfocado). \\
\hline Formación creativa (desenfocado). & Institución empleadora (enfocado). \\
\hline
\end{tabular}

Nota: en el nivel trabajo, la metaforización oculta y desenfoca algunos elementos propios del dominio original de la cultura. 
Con respecto a los modos de estructuración metafórica, existió una estructuración explícita y otra implícita. El primero ocurre cuando se halla en el discurso la enunciación directa de la metáfora, y el segundo, cuando si bien no se halla en el discurso la enunciación directa de la metáfora, sí se encuentran términos del léxico propio del dominio metafórico, es decir, se enuncia la metáfora de modo indirecto. Por ejemplo, el nivel metafórico "la cultura es trabajo" aparece en el corpus, pero son más las veces en las que se encuentra la estructuración implícita o estructuración léxica de dominio, esto es, por medio de términos del dominio metafórico, como son "empleados", "trabajo", "trabajadores", "remuneración", "jubilaciones", etcétera.

La metáfora funcionó como un mecanismo de representación discursiva, es decir, no como mero tropo a nivel frástico, sino como una estrategia del discurso con implicancias lingüísticas, textuales y discursivas, por ende, pragmáticas. En los textos analizados, esta figura convocó a los enunciadores de un modo peculiar a determinados roles y a cierta escenificación. Esto es, la metáfora funcionó también como un juego del lenguaje dramático y una proposición argumental.

\section{Las familias metafóricas y su valor como caracterizaciones}

La familia económica mantiene en su interior una coherencia antagónica: los niveles internos son temáticamente opuestos, incluso en relación dialéctica. La familia religiosa presenta una coherencia complementaria, debido a que sus diversos niveles forman parte del despliegue de una misma metáfora madre, por lo tanto, los niveles en el interior de la familia religiosa no son intercambiables.

En las familias metafóricas, cada uno de los niveles expande el dominio original y también dilata la capacidad de que, a partir de la estructuración del dominio original, se concrete una narración metafórica. Por ejemplo, la familia metafórica religiosa cuenta con varios niveles que habilitan ciertas acciones. El nivel templo implica acciones ligadas a acudir a un sitio con el objetivo de celebración, adoración u oración. El nivel celebración implica la acción conjunta de los fieles de celebrar alguna liturgia o ceremonia. Pero si se toman las acciones habilitadas por la suma de los niveles implicados en la familia metafórica religiosa, la capacidad dramática de la metaforización aumenta porque los núcleos narrativos latentes en cada dominio metafórico pueden conjugarse. Entonces, el uso -consciente o no- de una metáfora implica que la realidad sea narrada con ciertas posibilidades.

El hallazgo de metáforas referidas al patrimonio cultural, a los procesos de gestión y a la cultura en general implica el hallazgo de caracterizaciones contemporáneas de estos asuntos. Conviene recordar que por "discurso" no se comprende únicamente palabra sobre alguna cuestión u objeto, es decir, mera descripción, sino que, en la tradición de los estudios del discurso, este se concibe como una acción o conjunto de 
Felicitas Casillo

Caracterizaciones contemporáneas de la cultura: metáforas materialistas e idealistas acerca

del patrimonio cultural en textos online de La Nación, Clarín y Página/12

acciones del lenguaje y en el lenguaje, es decir, que el discurso convoca a los sujetos de un modo peculiar, los habilita a ciertos roles, los afecta de diversos modos.

En muchas oportunidades, los significados connotados en las metáforas son inasibles en un texto, forman parte de la vida abstracta del signo, y esto presenta el problema del origen de esos significados. Por ende, en este u otros estudios en los que solamente se cuenta con los textos, pero se los considera instancias de discurso, resultaría difícil describir los sistemas connotados. La solución hermenéutica de Paul Ricoeur (2001) supera esa dificultad: "Solo una respuesta es posible: es necesario tener en cuenta al oyente o al lector, y considerar la novedad de una significación emergente como la obra instantánea del lector" (p. 134).

\section{Familia metafórica económica}

La familia económica es aquel conjunto de metáforas temáticas en las que el rasgo mayormente destacado de la cultura es económico. Implica dos niveles: recurso y trabajo.

\section{Nivel recurso}

Ejemplos ${ }^{4}$

Clarín (20/11/2007): “En lo que el mercado produce solo [la industria cultural], el Estado no se tiene que meter. Nos interesa estimular lo que no se produce o no se consume [obras artísticas] espontáneamente".

Página/12 (24/5/2008): "El Colón siempre fue una fábrica de arte [...]".

La Nación (12/4/2009): “[...] el Colón estimuló la multiplicación de artistas de calidad [...], influencia [artística] que también derramó sus beneficios sobre la actividad teatral en general".

Página/12 (10/4/2010): “Como el negocio de romper todo para construir lo más barato posible, a costa del ecosistema cultural y social de nuestra ciudad, es muy grande, su influencia es también muy grande".

Página/12 (3/7/2010): "[...] diversas formas en que este recurso se transforma en un instrumento generador de valor".

La Nación (15/8/2010): “[...] comenzó una lenta declinación con algunos picos [estado del Teatro descrito a partir de la imagen de un gráfico económico]. Lo de ahora es una versión degradada de aquel modelo [programación del Teatro] porque cambió el panorama internacional. [...] Ahora no se puede repetir ese modelo sin replanteárselo".

\footnotetext{
En los ejemplos de las diferentes familias y niveles, la cursiva indica la presencia metafórica tanto explícita como implícita. Los corchetes fueron utilizados para aclarar la referencia de un término metafórico en el caso de que no quedara claro por la falta del "cotexto" del enunciado, es decir, por falta del conjunto de elementos lingüísticos que anteceden y siguen al término o enunciado metafórico.
} 
Austral Cmunicación

Volumen 9, número 2 (Diciembre de 2020): 159-186. ISSN 2313-9129

Clarín (31/8/2010): “Tenemos que entender que la cultura es una economía alternativa. [...] la alternativa es la cultura como modelo de desarrollo [...]".

El nivel recurso mantuvo un enfoque general economicista, en tanto procesos relacionados con la cultura se cuantifican y esta puede ser usada con ciertos fines de desarrollo. Este enfoque se concreta en las tres variaciones del nivel recurso: la variación empresarial, observable a partir de la estructuración léxica de dominio; la variación medioambiental, enunciada directamente y también de forma implícita a través del lexicón, y la variación industrial, también enunciada tanto directamente como a través del léxico de dominio.

La metáfora "la cultura es un recurso" implica la caracterización del patrimonio y la cultura desde una perspectiva económica, ligada a la economía y al desarrollo. Este nivel metafórico se concreta a partir de la metaforización explícita, es decir, la enunciación directa de la metáfora, o la metaforización implícita, a partir del uso de vocabulario ligado al ámbito empresarial, como puede ser: "potencializar", "producción", "oferta”, "puesta en valor", “promoción", "posicionamiento", "mercado”, "estimular", “consumir", “inversión”, “derramar beneficios”, “multiplicar”, “modelo", “minimizar pérdidas”, “optimizar", “progreso," “evolución”, “modernización”, "reciclaje”, “reestructuración”, “reordenamiento", "impacto económico", "modelo importador de producciones", "impacto urbano", "reactivación de centros urbanos" y "usos culturales que dinamicen", ente otros términos y construcciones observados en el corpus. A este tipo de metaforización se la llamó "variación empresarial", porque implica el uso de términos del management o del ámbito productivo.

El nivel metafórico recurso implica una intersección de un dominio económico y uno medioambiental: sugiere la comprensión de la cultura como una oportunidad semejante a la que representa la naturaleza para la economía. El término "recurso" es de uso común: alguna cosa en la naturaleza -un yacimiento, los bosques, el agua- es un recurso para la sociedad. Las connotaciones o "reverberaciones" -al decir de Lakoff y Johnson (2009, pp. 182-183) - de esta metáfora son problemáticas, porque, en tanto recursos, tienen una cotización y se agotan si la explotación es desmedida.

El uso de la construcción "recurso cultural", a partir del uso popular de "recurso natural", concibe al patrimonio como un elemento que permitirá el crecimiento económico y facilitará el desarrollo social. Sin embargo, por un lado, el uso de esta metáfora no explicita el peligro que sí se asocia al de la "explotación" de los recursos naturales y el peligro de que estos se "agoten". Por otro lado, los recursos naturales son un asunto recurrente y sensible en el espacio público. El uso de la intersección entre el dominio económico y el de la naturaleza para referirse a la cultura es al menos polémico, porque una de las implicaciones o "reverberaciones" de la metáfora es el riesgo del uso indiscriminado. También filosóficamente existió una crítica respecto al sometimiento de la 
Felicitas Casillo

Caracterizaciones contemporáneas de la cultura: metáforas materialistas e idealistas acerca

del patrimonio cultural en textos online de La Nación, Clarín y Página/12

naturaleza en el marco del capitalismo y esa misma crítica se extendió al ámbito de la cultura. Corrientes influyentes, como la Escuela de Frankfurt, se volvieron contra este efecto de dominio de la Ilustración, y fue dentro del paradigma crítico que surgió la denuncia sobre la industria cultural. Se anticipó, de esta manera, el uso exclusivamente económico como un sometimiento de la cultura semejante al sometimiento de la economía sobre la naturaleza.

El nivel metafórico recurso, entonces, destaca la oportunidad que significa la actividad cultural, pero desenfoca el riesgo de la "sobreexplotación" del recurso y oculta otros elementos supuestos del dominio cultura, como la educación artística, la labor creativa o las motivaciones artísticas. Por ende, la familia metafórica económica implica que elementos del propio dominio metafórico aparezcan desenfocados, pero que "reverberen" y que sea posible asociarlos semánticamente a la metáfora. Esas asociaciones, esos nudos dramáticos latentes habitan el dominio de la metáfora y pueden activarse por el oyente o lector.

Otra crítica posible al uso de este tipo de discurso consiste en que la concepción de cultura como recurso podría connotar que la cultura es algo dado, perteneciente al ecosistema o medioambiente, algo por transformar. La noción de "puesta en valor", por ejemplo, es cercana semánticamente a las de "valor agregado" o "cadena de valor". Sin embargo, la cultura es labor humana y no contexto natural: posee ya significados para una comunidad de sentido. Un edificio patrimonial, por ejemplo, incluso en estado de ruinas, es en sí mismo valioso y, debido a ese valor, merece ser restaurado.

La referencia a la cultura del nivel recurso, entonces, se concreta por medio del vocabulario ligado a la cuantificación y se asocia a la noción de "uso" de la cultura con cierto fin. La connotación semántica de recursos naturales/explotación/necesidad de preservación podría ser una inferencia discursiva compleja y no deseada por los enunciadores. $\mathrm{Si}$ además se tiene en cuenta la significativa injerencia del campo político en la aparición de esta metáfora, su uso podría resultar restrictivo para referirse a la cultura y esto podría provocar posiciones casi inconciliables entre gestores, creadores y audiencia.

Una de las variaciones metafóricas del nivel recurso fue la de "cultura es un ecosistema". La variación "ecosistema cultural" compara directamente la cultura con la naturaleza. El término "ecosistema" es utilizado en ciencias sociales para definir diversas realidades en las que existen elementos interrelacionados. El riesgo de este tipo de discurso consiste en limitar una realidad social al discurso de las ciencias biológicas, en las que existen tópicos fuertes, como podrían ser la subsistencia de los individuos o especies fuertes, la adaptación o la evolución natural.

La tercera variación de este nivel es la industrial, menos abstracta, aunque también económica y cercana al nivel trabajo. La metaforización suele ser explícita, ligada al ámbito industrial e incluso mecánico, por ejemplo, el teatro como una "fábrica de arte". 
Austral Cmunicación

Volumen 9, número 2 (Diciembre de 2020): 159-186. ISSN 2313-9129

\section{Nivel trabajo}

Ejemplos

Página/12 (6/6/2008): “Bajo el título 'Defendamos al Teatro Colón', los trabajadores del primer coliseo le reclamaron al gobierno que encabeza Mauricio Macri [...]”.

La Nación (13/2/2008): “[...] y no descartó que se puedan producir despidos de gente que 'no trabaja', entre los más de 1.300 empleados que actualmente tiene el teatro".

Página/12 (5/11/2010): “[...] el maltrato sistemático a las compañeras y compañeros del Ballet Estable; y la falta de respuesta de la autoridad sobre la implementación de las mínimas condiciones dignas de trabajo".

Clarín (17/2/2011): "En una asamblea realizada ayer, trabajadores del Teatro Colón resolvieron continuar con el cese de actividades con presencia en el lugar de trabajo declarado antes de fin de 2010, y pusieron en duda el inicio de la temporada 2011".

La Nación (20/1/2011): "Ibarra precisó que el convenio consta de dos ejes: uno relacionado con un 'plan de carrera para los trabajadores'; y otro, basado en una mejora salarial, que 'prevé un concepto de productividad que está asociado al pleno funcionamiento del teatro"'.

El nivel trabajo, opuesto al nivel recurso, mantiene un enfoque crítico. El paradigma crítico había sustituido el conflicto de lucha de clases como motor de la historia por el de lucha del hombre versus naturaleza, y había sugerido que uno de los efectos de la Ilustración era también el sometimiento de la cultura. Este nivel metafórico caracteriza a la institución cultural únicamente como fuente laboral.

Frente al anterior nivel metafórico, la metáfora "la cultura es trabajo" se concreta sobre todo a partir de la estructura de dominio implícito o léxico y a través de un discurso dialéctico, que opone "trabajadores" y "dirigentes". Ejemplos de metaforización por medio del léxico del dominio son "trabajadores del teatro", "las plantas estables del teatro", "achicamiento", "estabilidad laboral", "empleados", "sindicato de músicos", "trabajadores especializados", “mínimas condiciones dignas de trabajo", “asambleas", “cese de actividades con presencia en el lugar de trabajo", "plan de carrera para los trabajadores", "concepto de productividad", "cesanteados", etcétera.

Este nivel consiste en una metonimia de funcionamiento metafórico en tanto se asume que la condición de trabajador del artista es el rol determinante en el debate público acerca de instituciones ligadas al patrimonio cultural. Sin embargo, el funcionamiento metafórico de esta metonimia consiste en que la semejanza que se establece entre las dos gestalts o dominios es estructural, es decir, la estructura de la cultura aparece exclusivamente organizada y enunciada a través de la estructura del trabajo. De esta manera, el Teatro aparece mencionado como "lugar de trabajo", donde deben darse unas "condiciones de trabajo", pero no se especifica en qué consiste la particularidad de esa labor o cuáles son esas condiciones. Es decir, la mención de la cultura como trabajo y el artista 
exclusivamente como trabajador se transforma en metáfora estructural en tanto se adopta la estructura referida al dominio laboral o al dominio de protesta o reclamo laboral, y permanece oculta o desenfocada la peculiaridad de la labor artística. "Trabajadores" o "empleados" son, finalmente, términos válidos para cualquier ámbito de producción.

La estructura de dominio metafórico implica que los artistas sean denominados como "trabajadores" y "empleados", asociados a la actividad gremial de los sindicatos Asociación Trabajadores del Estado (ATE) y Sindicato Único de Trabajadores y Empleados de la Ciudad de Buenos Aires (SUTECBA). A su vez, aparecen relacionados con asuntos salariales, contrataciones, condiciones laborales, protestas, pero solamente de modo excepcional se mencionan reclamos artísticos o ligados a la performance o educación. Es decir, este nivel destaca aquellas dimensiones laborales de la cultura, pero desenfoca aquellas exclusivamente artísticas y oculta la creación, la experiencia de la audiencia, las motivaciones no económicas y la formación.

Tanto el nivel recurso como el nivel trabajo no escapan de una lógica exclusivamente economicista sobre cultura. De esta manera, la cultura permanece cautiva en un discurso "de pinzas", en el que no se subraya qué define realmente al campo cultural. Mientras que el nivel trabajo mantiene la retórica de denuncia propia de movimientos y corrientes críticos hacia el capitalismo, no parece que imponer un discurso exclusivamente económico o productivo haya solucionado los problemas. Por el contrario, la lógica de la producción sintetiza rápidamente ese vocabulario, como sucedió con la normalización del término "industria cultural", y se afianza un discurso solamente productivo sobre la cultura.

Es necesario aclarar que por medio de este análisis no se pretende negar dificultades relacionadas al empleo que puedan tener los artistas, o a cuestiones del derecho laboral, como son jubilaciones y contratos, tampoco se pretende dar a entender que el ser artista es una condición superior a la de un trabajador de la seguridad, un sastre o un mecánico. Lo que se intenta sugerir es que, en el contexto del caso estudiado, los problemas referidos al Teatro no eran únicamente laborales, sino que debían resolverse cuestiones artísticas y edilicias, entre otras, además de las referidas al régimen laboral. Esos problemas aparecen desenfocados por un discurso economicista.

El nivel metafórico "la cultura es trabajo" no presenta variaciones y a menudo asume enunciados o términos de larga trayectoria ideológica, como son "cese de actividades en el lugar de trabajo", "asambleas", "piqueteros", "amedrentamiento", entre otras. También aparece el colectivo de identificación -en términos de Eliseo Verón (1985) kirchnerista "las compañeras y los compañeros" para referirse a colegas.

\section{Familia metafórica religiosa}

Esta familia es deudora de una definición idealista, posiblemente común en el contexto histórico original de la institución que protagonizó este análisis. Esta caracteri- 
Austral Comunicación

Volumen 9, número 2 (Diciembre de 2020): 159-186. ISSN 2313-9129

zación se concreta en diversos niveles que enuncian el campo cultural como territorio ligado a lo sobrenatural y a la sacralidad del arte, a partir de una lectura emotiva y nostálgica del patrimonio y de la cultura. Particularmente, en el caso del conflicto del Teatro Colón, se acentúa este efecto debido a la coyuntura del Bicentenario patrio, celebrado en la República Argentina en 2010.

En la mayoría de los enunciados en los que aparece la conceptualización de estas metáforas, el tono es opuesto al de la familia económica. Si en la primera familia el dominio se relacionaba con la gestión y el trabajo, con la cuantificación y los reclamos, en la familia religiosa el dominio se relaciona con el inasible mundo místico de los valores culturales de una nación. Sin embargo, no siempre sucede esto: por ejemplo, cuando se subraya a la cultura como recurso para el desarrollo (en la familia económica), en ocasiones se enuncia también la necesaria participación de todos o la pertenencia de la institución cultural a la ciudadanía (familia religiosa, nivel celebración). Hay entre estos niveles metafóricos -nivel recurso y nivel celebración-, una posible coherencia. Es decir, entre el mundo posible que alberga cada metáfora -los núcleos narrativos que habilita, los sujetos que convoca al discurso y las características que subraya- existe acceso en más de una oportunidad.

La familia religiosa suele estar ligada a un lugar común sobre la cultura, en el sentido de que se refiere a la realidad cultural desde estereotipos poco precisos y alusiones difusas sobre el mito de origen de la cultura en cuestión. En la metáfora económica, los actores presentados eran funcionarios ligados a la dirección, a la gestión o al trabajo; en el caso de la metáfora religiosa, la cultura se representa como una liturgia a celebrar en un templo frente a una multitud de fieles. Los actores presentados por fuera de la audiencia son afamados artistas que aparecen en un rol sacerdotal, como pueden ser los casos de Daniel Barenboim, Mercedes Sosa, Plácido Domingo o Paloma Herrera. Sin embargo, no aparecen en esta metáfora los artistas de menor prestigio y fama, que son mayoría entre los grupos de bailarines, los coros y las orquestas.

\section{Nivel celebración}

Ejemplos

La Nación (19/12/2007): "No habrá figuras de la política internacional que aplaudan de pie la gran celebración de nuestra cultura ni habrá imágenes que reproduzcan un glorioso momento [...]".

Clarín (22/4/2008): "La frase que se utilizará para finalizar las acciones sintetizará el espíritu a modo de mensaje: Teatro Colón. Es argentino, cumple 100 años y es de todos".

Clarín (25/5/2010):““Vení al Colón, es de todos’ recomendaba la voz de Maximiliano Guerra”. 
Felicitas Casillo

Caracterizaciones contemporáneas de la cultura: metáforas materialistas e idealistas acerca

del patrimonio cultural en textos online de La Nación, Clarín y Página/12

La Nación (25/5/2010): "No es de Macri ni de la Presidenta, sino de todos".

Clarín (27/5/2010): "Las fiestas son sagradas para los pueblos, y el que pretende usar y apoderarse de esas fiestas es castigado por los dioses; así es el viejo mito de la antigüedad".

Página/12 (23/03/2011): "La información fue confirmada por el ministro de Cultura de la Ciudad, Hernán Lombardi, quien lamentó que el espectáculo coincida con las movilizaciones por el Día de la Memoria, pero arriesgó que el encuentro será 'una fiesta de la cultura".

En el nivel celebración se considera a la cultura como una oportunidad para la fiesta o la celebración y se subraya el acceso de "todos" a ella. El nivel emerge en algunas ocasiones desde el campo político, y en esas ocasiones se lo podría vincular al nivel recurso de la familia económica, en el sentido de que para que la cultura realmente sea un recurso para el desarrollo, requeriría de una participación masiva o, al menos, no restringida.

El nivel celebración de la familia religiosa presenta una variación cívica. Esta es muy semejante a la enunciación normal del nivel celebración, pero connota no un valor religioso, sino un valor cívico asociado a la cultura como valor democrático o republicano. Algunos ejemplos de esta variación son: "devolución del Teatro a la ciudadanía" "el teatro Colón volvió a ser de los vecinos", "(el Teatro produjo) momentos de comunión colectiva”.

\section{Nivel símbolo}

Ejemplos

Clarín (20/2/2008): "El Teatro Colón es un símbolo de la cultura argentina, realizado, cuidado y preservado con gran esfuerzo y orgullo".

La Nación (23/5/2008): “[...] el Colón es una radiografía del país”.

La Nación (25/5/2008): "El signo de distinción del imaginario social argentino".

Página/12 (7/6/2008): "Que el Teatro Colón no esté funcionando es un indicio de que las cosas no funcionan".

Página/12 (16/1/2009): “Macri no le da sentido al patrimonio histórico. La Ciudad tiene que tomar una decisión del sentido que tiene que tener el Colón: si será un espacio frívolo o va a ser el gran coliseo que siempre fue, dijo la líder de la Coalición Cívica, Elisa Carrió".

La Nación (15/2/2009): “El altísimo (e imprescindible) valor emblemático que irradia el Teatro Colón sobre toda la cultura argentina (no únicamente sobre quienes lo frecuentan) $[\ldots]$..

Clarín (12/5/2010): “Dada la compleja dimensión simbólica que proyecta -puertas adentro y puertas afuera- en el campo de las ideas, las costumbres, el gusto, la política, 
Austral Comunicación

Volumen 9, número 2 (Diciembre de 2020): 159-186. ISSN 2313-9129

el Teatro Colón no es un venerable objeto de estimación y de crítica únicamente para sus afortunados habitués, sino también para la sociedad en su conjunto".

Página/12 (27/5/2010): "En el Teatro Colón reunieron a la Argentina del pasado: todos fósiles, vejestorios, con la presencia de (Ricardo) Fort y la estética de los noventa".

Mientras que el nivel celebración subrayaba el acceso o experiencia de la audiencia, este nivel subraya el significado del patrimonio: un espíritu o una tradición artística ligados a una gloria pasada significativa que debe actualizarse, salvarse, renovarse, refundarse o recuperarse. "La cultura -o la institución patrimonial- es un símbolo", fue la enunciación directa de una metonimia de funcionamiento metafórico, semejante al caso del nivel trabajo de la familia económica. Es decir, la estructuración metafórica ocurre no a partir de la relación con otro dominio, sino con la elección de un rasgo propio de la cultura -su valor simbólico- y la estructuración metafórica a partir de ese rasgo. El dominio del nivel simbólico consiste en las características de un símbolo y las acciones que pueden realizarse sobre un símbolo.

A pesar de que el valor simbólico de la cultura podría estar ligado a la vanguardia o a la innovación, dependiendo de la institución u objeto artístico que protagonice en la conversación pública, en el caso estudiado se relaciona con el valor del patrimonio en relación con el pasado: aquello que fue es lo que otorga valor a lo que hoy es y debe recuperarse. El nivel metafórico símbolo estructura el dominio cultural en el sentido de que concibe a la cultura exclusivamente como el emblema de algo: una etapa de la historia argentina, la excelencia, el arte, etc. Como la familia religiosa en general, este nivel implica también una fuerte apelación a la emotividad.

Las variaciones que aparecen son sinonímicas, es decir, sinónimos -términos intercambiables que aluden un mismo significado- de "símbolo", como son "emblema", "indicio", "ícono", "marca" o, incluso, "radiografía". La metaforización en el nivel simbólico se produce tanto de forma directa, a partir de la enunciación explícita de la metáfora, o de forma indirecta, a partir de la metaforización implícita o léxica, esto es, cuando se subraya el significado del Teatro, su valor patrimonial como gloria pasada, sofisticación, etcétera.

\section{Nivel templo}

Ejemplos

La Nación (22/5/2008): “[...] o el valor agregado de templo artístico que acompañó desde siempre al teatro".

Página/12 (24/5/2008): "Mañana será la fecha más esperada de la liturgia operística argentina”.

Página/12 (24/5/2008): "Y el Colón es un tesoro ahora cerrado, atrapado en una obra demorada a la mejor manera argentina, cuestionada y cuestionable". 
Felicitas Casillo

Caracterizaciones contemporáneas de la cultura: metáforas materialistas e idealistas acerca

del patrimonio cultural en textos online de La Nación, Clarín y Página/12

La Nación (25/5/2008): "Vale recordar que estamos en la Argentina, país con una buena parte de su cultura musical modelada por la inmigración italiana, devota de la ópera. Por lo tanto, llegar al Colón ha sido, en cierta medida, llegar al espacio sagrado de ese canto lírico que atravesó distintas clases sociales con cierto efecto integrador. De ahí la identificación del Estado argentino con el Colón: la institucionalización de la música, pero también el espectáculo musical como ritual de la Nación".

Página/12 (10/10/2009): “Decidir si el magnífico teatro está en peligro mucho, poquito o nada ya es tarea de teólogos [...]".

La Nación (22/12/2009): "[...] el edificio considerado la joya del patrimonio y una meca para los melómanos del mundo entero".

La Nación (22/5/2010): "Peor sería imaginar una batalla simbólica sobre la construcción de un altar de la patria. Menos mal que ese tipo de 'lugares de memoria' ha caído en desuso".

Clarín (24/5/2010): “[...] proteger su acústica, venerada en todo el mundo".

La Nación (15/8/2010): “[...] el Colón es el símbolo más grande. Si el teatro encuentra la cuadratura del círculo y se proyecta va a dejar de ser la catedral para transformarse en algo mucho más dinámico".

La enunciación del nivel templo suele ser directa y consiste en "el Teatro es un templo". Allí sucedería una liturgia o acontecimiento sobrenatural. Algunos enunciados se categorizaron como nivel templo variación tesoro, y, en ese caso, "el Teatro es una joya" o "el Teatro es un tesoro" que debe ser defendido y salvaguardado; por ejemplo, "el edificio considerado la joya del patrimonio y una meca para los melómanos del mundo entero", en una noticia publicada el 22 de diciembre de 2009 en el diario La Nación. Ambas metáforas, si bien son distintas, pertenecen a la misma asociación de cultura como valor extraordinario y confieren excepcionalidad a la institución. Otra variación observada es la del nivel templo variación cívica, que funciona de forma semejante a la de variación cívica del nivel celebración. Por ejemplo: "la construcción de un altar de la patria", en una noticia publicada el 22 de mayo de 2010 en el diario La Nación.

\section{Nivel sacerdotal}

Ejemplos ${ }^{5}$

Clarín (25/5/2008): “Una Argentina bastante necesitada de Barenboims".

La Nación (29/5/2008): “[...] -el mayor genio musical de la actualidad dirigiendo uno de los organismos sinfónicos más distinguidos del mundo-, con un concierto

\footnotetext{
En algunos casos del nivel sacerdotal, la cursiva señala el enunciado completo porque el rol desde el cual enuncia el artista es sacerdotal. Es decir, se trata de metáforas discursivas que se concretan a partir de un nivel pragmático y no únicamente por la referencia de los términos y construcciones.
} 
Austral Cmunicación

Volumen 9, número 2 (Diciembre de 2020): 159-186. ISSN 2313-9129

extraordinario el $1^{\circ}$ de junio en el Luna Park, destinado a convertirse en el mayor homenaje que se le tributará al Teatro Colón en su centenario".

La Nación (7/6/2008): "(Daniel Barenboim dixit) '[...] Por eso apelo a todas las personas responsables, irresponsables de esta situación para que se olviden de los orgullos $y$ de las ambiciones personales porque todo eso es muy pequeño; el gran orgullo es que el Teatro se abra de nuevo para el Bicentenario, en 2010. Nuestro Himno Nacional habla de los laureles que supimos conseguir. Los laureles, señoras y señores, no son eternos, hay que reinventarlos todos los días".

La Nación (23/5/2010): "(Paloma Herrera dixit) 'en esta nueva etapa del Teatro Colón debemos renovar la esperanza de devolverle su destino glorioso".

Clarín (22/0/2011): "(Plácido Domingo dixit) 'Comprendo a las dos partes. Desgraciadamente los que sufren con esto son los inocentes: el público. Es lo que esperamos poder salvar"'.

El nivel sacerdotal no fue enunciado de manera explícita, en cambio, artistas de fama y prestigio son enunciados en el discurso con un rol ligado a la autoridad moral, como mediadores o celebrantes de la cultura. También se observó una variación cívica del nivel sacerdotal, cuando su rol se relaciona con la nación o la democracia y sin una connotación religiosa. Por ejemplo: "una Argentina bastante necesitada de Barenboims", en una noticia publicada el 25 de mayo de 2008 en Clarín.

Posiblemente, este nivel carecería de fuerza si no existiera una familia metafórica que pudiera sostener su sentido. En la familia religiosa, cada nivel representa un elemento del dominio, y es razonable que el nivel sacerdotal sea inferido por el lector a partir de los otros niveles. Se ha llamado a estas metáforas como "estructurales discursivas" porque suceden cuando la enunciación mediática habilita a un actor relacionándolo con cierto rol o porque un actor determinado enuncia a partir de un rol.

La metáfora sacerdotal de la familia religiosa funcionó de dos modos distintos: en el primero, el medio describe a cierto actor con características del rol sacerdotal; en el segundo, el actor asume el rol sacerdotal, es decir, el enunciado suele ser una prescripción acerca de qué debe hacerse.

\section{Conclusiones}

Las caracterizaciones halladas en el corpus, descritas a partir del concepto de "familias metafóricas", no pueden generalizarse por fuera del caso de estudio. En cambio, estas descripciones contribuyen a la construcción de hipótesis o variables que otras investigaciones futuras podrían abordar.

Como se expuso al comienzo de este artículo, durante la modernidad se han ido alternando la visión idealista sobre la cultura, que propiciaba la valoración espiritual, y la visión marxista, que propiciaba la determinación economicista. Esas caracterizacio- 
Felicitas Casillo

Caracterizaciones contemporáneas de la cultura: metáforas materialistas e idealistas acerca

del patrimonio cultural en textos online de La Nación, Clarín y Página/12

nes coexistieron, a pesar de que en diversos periodos una $\mathrm{u}$ otra hayan tenido mayor influencia. La característica de estas definiciones de "cultura" consistía en que subrayaban un único factor como determinante del hecho cultural: un factor espiritual o un factor material. Dempf (1933) consideraba que la importancia de las concepciones de la cultura consistía en que, siendo falsas o ciertas, estas tenían "eficacia histórica" y que "toda concepción de la cultura es un espejo en el que se refleja la constelación entera de la realidad vital" (p. 102).

El hallazgo de familias metafóricas acerca del patrimonio implica que la consideración social de este ocurre en los límites con otros ámbitos o áreas. La familia religiosa sugiere que esos sitios son tan importantes para las sociedades contemporáneas como los templos y lugares sacros. La familia económica sugiere la tensión que atraviesan las instituciones patrimoniales con respecto a la solvencia y describe tanto requerimientos laborales como institucionales y estatales.

La familia religiosa de metáforas mantuvo muchas de las características de la definición idealista. En el contexto del Bicentenario, este discurso sobre la cultura pareció acentuarse: diversas personalidades y partidos políticos recurrieron a las significaciones del patrimonio como una forma de legitimación. Por ejemplo, cuando se alude que el patrimonio "es de todos", que debe "celebrarse", que el Colón es un "símbolo" de lo que debe volver a ser la Argentina, entre otros. El patrimonio, entonces, pareció funcionar como un repertorio de sentido, eficaz para las siempre ávidas narrativas políticas.

La familia de metáforas económicas, por su parte, tampoco es una caracterización novedosa en la historia de la cultura, pero sí lo son ciertas tendencias descritas dentro de esta familia de metáforas. Los dos niveles de la familia económica son antagónicos, en relación dialéctica: el nivel recurso y el nivel trabajo.

El nivel recurso, por un lado, parece el más complejo, en tanto existió una enunciación directa, "la cultura y el patrimonio son un recurso"; una variación empresarial en la que la cultura era planificada en términos del management, y una variación medioambiental, que podría responder al vínculo descripto por Dempf (1933) entre liberalismo económico y darwinismo (por ejemplo: "[el Teatro como parte del] ecosistema cultural”). Esta relación resulta problemática porque, además de ocultar y desenfocar la realidad propia del campo cultural, asociar cultura y naturaleza implica una concepción ingenua acerca de lo cultural como algo dado y asocia a la cultura con la riesgosa industrialización de la naturaleza. Por otro lado, el nivel trabajo concibe a los artistas como "trabajadores" y al Teatro como una "fábrica", mientras que el discurso gira en torno a reclamos "en el lugar de trabajo" por "sueldos", "ajustes" y "despidos". Este nivel es más sencillo, en el sentido de que implicó un planteo típico del folklore socialista. 
En el contexto actual, mientras que el capitalismo y la política se revisten de discursos de corte estético-publicitario, es sugerente que el discurso ligado a la gestión de la cultura se vuelva, por el contrario, árido y excesivamente empresarial. A pesar de que la gestión y la solvencia son asuntos cruciales dentro del campo cultural, parece sesgado el uso de metáforas que ocultan particularidades de una institución cultural y de una actividad artística. Por ejemplo: "[el Teatro] estimula la multiplicación de artistas", "derrama beneficios" o es “instrumento generador de valor". El origen de este fenómeno de cambio discursivo excede el presente estudio, aunque sí es posible aventurar un derrotero histórico de este fenómeno por demás interesante.

Resulta al menos acotado caracterizar a la cultura como producto meramente industrial, sometida a una lógica de mediciones y rentabilidad, y también lo es caracterizarla simplemente como fuente de trabajo. En el marco teórico de este artículo, se ha hecho referencia también al problema de la definición performativa del patrimonio que suele sustentar algunas de las caracterizaciones economicistas ligadas a la gestión cultural. Estas críticas se sostienen desde una perspectiva hermenéutica de la cultura (Ablett y Dyer, 2009; Gadamer, 1996; Morisset, 2011; Steiner, 1993; Tilden, 1977). En línea con este enfoque, la metodología aplicada se basó en la propuesta de Paul Ricoeur (2001) acerca de una "significación emergente" aportada por el lector (p. 134), sumada a la sugerencia de Umberto Eco (1992) de "fijar ciertos límites" a la interpretación (p. 85). Esta suponía siempre un diálogo; en palabras de George Steiner (1993): "La hermenéutica como puesta en acto de un entendimiento responsable" (p. 19). En este sentido, la misma metodología diseñada para el presente trabajo -unas etapas de lectura sucesivas y circulares- ensayó en el corpus un movimiento interpretativo.

Con respecto a las metaforizaciones halladas y descriptas, es necesario señalar que en el ámbito cultural este juego del lenguaje implica algunas particularidades. La cultura es ya en sí misma una actividad simbólica compleja. Al decir de Eco (1992), las obras funcionan como "metáforas epistemológicas" de una época (p. 40). Por ende, decir que la cultura o el patrimonio "son otra cosa" implica una lógica que se podría denominar de "metametáfora". Si la metáfora tuvo, además de su valor poético, un valor retórico explicativo, cabría preguntarse si este tipo de comparaciones fueron explicativamente fértiles en la conversación pública acerca de la cultura.

Es necesario subrayar, finalmente, que la comunidad de sentido en el campo cultural no está integrada solo por actores e instituciones presentes, sino que implica una tradición que abriga, a su vez, trascendencia futura. A través de este intercambio, podrían intentarse la comprensión de la cultura y el diseño de una gestión respetuosa tanto de legados físicos como intangibles. Este esfuerzo es la clave de una perspectiva hermenéutica, que asume la interpretación de objetos y asuntos patrimoniales o culturales a partir del contexto en el que se hallan y de los sentidos que les han sido dados. 
Felicitas Casillo

Caracterizaciones contemporáneas de la cultura: metáforas materialistas e idealistas acerca

del patrimonio cultural en textos online de La Nación, Clarín y Página/12

\section{Referencias}

Ablett, P. G. y Dyer, P. K. (2009). Heritage and hermeneutics: Towards a boarder interpretation of interpretation. En Current Issues in Tourism (Vol. 12, pp. 209233). Maroochydore: Routledge.

Adorno, T. y Horkheimer, M. (1998). Dialéctica de la Ilustración. Madrid: Editorial Trotta.

Angenot, M. (2010). El discurso social. Buenos Aires: Siglo XXI.

Aristóteles. (1948). El arte poética. Buenos Aires: Espasa-Calpe.

Arnold, M. (2010). Cultura y anarquía. Madrid: Ediciones Cátedra.

Austin, J. L. (1962). Cómo hacer cosas con las palabras. Barcelona: Paidós.

Baldwin, J. R., Faulkner, S. L., Hecht, M. L. y Lindsley, S. L. (2008). Redefining Culture. Perspectives Across the Disciplines. Nueva Jersey: Lawrence Erlbaum Associates.

Ballart, J. (1997). El patrimonio histórico y arqueológico. Valor y uso. Barcelona: Ariel.

Benjamin, W. (2015). La obra de arte en la era de la reproductibilidad técnica y otros ensayos. Buenos Aires: Ediciones Godot.

Bourdieu, P. (2010). El sentido social del gusto. Elementos para una sociología de la cultura. Buenos Aires: Siglo XXI.

Buitrago Restrepo,F.y Duque Márquez,I.(2013).La economía naranja. Una oportunidad infinita. Nueva York: BID/Aguilar.

Dempf, A. (1933). Filosofía de la cultura. Madrid: Editorial Revista de Occidente.

Dormaels, M. (2011). Patrimonio, patrimonialización e identidad. Hacia una hermenéutica del patriomonio. Revista Herencia, 24, 7-14.

Du Gay, P. y Pryke, M. (2002). Cultural Economy. Londres: Sage Publications.

Eagleton, T. (1999). La función de la crítica. Barcelona: Ediciones Paidós.

Eagleton, T. (2000). The Idea of Culture. Oxford: Blackwell Publishing.

Eco, U. (1992). Obra abierta. Barcelona: Planeta.

Eliot, T. S. (1948). Notes towards the Definition of Culture. Londres: Faber and Faber Limited.

Fauconnier, G. (1997). Mapping in thought and language. Nueva York: Cambridge University Press.

Fernández Pedemonte, D. (1996). La producción de sentido en el discurso poético. Buenos Aires: Edicial.

Fernández Pedemonte, D. (1999). Diarios y empresas: relatos de conflictos. Buenos Aires: Facultad de Ciencias de la Comunicación, Universidad Austral.

Fernández Pedemonte, D. (2011). La guerra por las representaciones. Mediatización y disenso en el gobierno de Cristina Fernández de Kirchner. En Elizalde, L. Fernández Pedemonte, D. y Riorda, M. (eds.). La gestión del disenso. Buenos Aires: La Crujía.

Gadamer, H. G. (1996). La actualidad de lo bello. Barcelona: Paidós. 
Austral Comunicación

Volumen 9, número 2 (Diciembre de 2020): 159-186. ISSN 2313-9129

García Canclini, N. (1987). Políticas culturales en América Latina. Distrito Federal: Grijalbo.

García Canclini, N. (1989). Cultura y sociedad. Distrito Federal: Cuaderpol SEP.

García Canclini, N. (1997). Cultura y comunicación: entre lo global y lo local. La Plata: Facultad de Periodismo y Comunicación Social, Universidad Nacional de la Plata. García Canclini, N. (1999). Los usos sociales del Patrimonio Cultural. En Aguilar Criado, E. Patrimonio Etnológico. Nuevas perspectivas de estudio (pp. 16-33). Andalucía: Consejería de Cultura, Junta de Andalucía.

García Canclini, N. (2010). La sociedad sin relato. Buenos Aires: Katz.

García Canclini, N. (2012). Cultura y desarrollo. Buenos Aires: Paidós.

Gibbs, R. (1994). The poetics of mind: Figurative thought, language, and understanding. Cambridge: Cambridge University Press.

Glucksberg, S. (2001). Understanding figurative language. Oxford: Oxford University Press.

Grady, J., Oakley, T. y Coulson, S. (1999). Blending and metaphor. En Steen, G. y Gibbs, R. (eds.). Metaphor in cognitive linguistics. Filadelfia: John Benjamins.

Gramsci, A. (2017). Antología. Selección y traducción de Manuel Sacristán. Barcelona: Siglo XXI.

Groys, B. (2008). Bajo sospecha. Una fenomenología de los medios. Valencia: Pre-textos. Gombrich, E. (2015). Breve historia de la cultura. Barcelona: Ediciones Península.

Hopkins, J. (2013). The creative economy. How people make money from ideas. Londres: Penguin.

Jahoda, G. (2012). Critical reflections on some recent definitions of 'culture'. Culture y Psychology, (18), 289-303.

Johnson, M. (1981). Philosophical perspectives on metaphor. Mineápolis: University of Minnesota Press.

Kövecses, Z. (2002). Metaphor: A practical introduction. Oxford: Oxford University Press.

Kroeber, A. L. y Kluckhohn, C. (1952). Culture: A Critical Review of Concepts and Definitions. Cambridge: Peabody Museum of American Archeology.

Lakoff, G. y Johnson, M. (2009). Metáforas de la vida cotidiana. Madrid: Cátedra Teorema.

Lakoff, G. (1987). Woman, Fire and Dangerous Things. What categories reveal about the Mind. Chicago: The University of Chicago Press.

Lakoff, G. (1994). The contemporary Theory of Metaphor. California: California Digital Librery.

Lakoff, G. (2002). Moral politics. How liberals and conservatives think. Chicago: The University of Chicago Press. 
Felicitas Casillo

Caracterizaciones contemporáneas de la cultura: metáforas materialistas e idealistas acerca

del patrimonio cultural en textos online de La Nación, Clarín y Página/12

Lakoff, G. y Turner, M. (1989). More than Cool Reason: A Field Guide to Poetic Metaphor. Chicago: The University of Chicago Press.

Lipovetsky, G. y Serroy, J. (2015). La estetización del mundo. Vivir en la época del capitalismo artístico. Buenos Aires: Anagrama.

Mironenko, I. A. y Sorokin, P. S. (2018). Seeking for the Definition of "Culture": Current Concerns and their Implications. A Comment on Gustav Jahoda's Article "Critical Reflections on some Recent Definitions of "Culture. Integrative Psychological and Behavioral Science, (52), 331-340. https://doi.org/10.1007/s12124-018-9425-y.

Morisset, L. K. (2011). Por una hermenéutica de las formas urbanas. En Morisset, L. K. y Breton, M.E. (dirs.). La villa, fenómeno de representación. Coll. Patrimoine urbain, (5). Québec: Presses de l'Université du Quebéc.

Pernoud, R. (2010). Para acabar con la edad media. Barcelona: Medievalia.

Resch, C. y Steinert, H. (2003). Industria cultural: conflictos en torno a los medios de producción de la clase culta. En Modelle kritischer Gesellschaftstheorie: Traditionen und Perspektiven der Kritischen Theorie (pp. 312-339). Stuttgart: Metzler'sche Verlagsbuchhandlung y Carl Ernst Poeschel Verlag GmbH.

Ricoeur, P. (2001). La metáfora viva. Madrid: Ediciones Cristiandad.

Rosaldo, I. R. (2008). Definiendo cultura. En Baldwin, J. R., Faulkner, S. L., Hecht, M. L. y Lindsley, S. L., Redefining Culture. Perspectives Across the Disciplines. Nueva Jersey: Lawrence Erlbaum Associates.

Searle, J. (1979).Expression and meaning: Studies in the theory of speech acts. Cambridge: Cambridge University Press.

Semino, E. (1997). Language and world creation in poems and other texts. Londres: Longman.

Steiner, G. (1993). Presencias reales. Buenos Aires: Destino.

Tilden, F. (1977). Interpreting our Heritage. Chapell Hill: The University of North Carolina Press.

Vasilachis de Gialdino, I. (2006). Estrategias de investigación cualitativa. Barcelona: Gedisa.

Verón, E. (1985). El discurso político. Buenos Aires: Hachette.

Williams, R. (1953). The idea of culture. Essays in Criticism, III(3), 239-266. https://doi. org/10.1093/eic/III.3.239.

Williams, R. (1981). Sociología de la cultura. Barcelona: Paidós.

Wittgenstein, L. (1953). Philosophical Investigations. Oxford: Basil Backwell.

Yúdice, G. (2002). El recurso de la cultura. Barcelona: Gedisa. 